\title{
Safe Joint Mechanism using torsion springs for Collision Safety and Positioning Accuracy of a Robot Arm
}

\author{
Adel Belharet, Jae-Bok Song ${ }^{\dagger}$ \\ Mechanical Engineering, Korea University
}

\begin{abstract}
In recent years, the potential for collision between humans and robots has drawn much attention since service robots are increasingly being used in the human environment. A safe robot arm can be achieved using either an active or passive compliance method. A passive compliance system composed of purely mechanical elements often provides faster and more reliable responses to dynamic collision than an active system involving sensors and actuators. Since positioning accuracy and collision safety of a robot arm are equally important, a robot arm should have very low stiffness when subjected to a collision force capable of causing human injury. Otherwise, it should maintain a very high stiffness. To implement these requirements, a novel safe joint mechanism (SJM-IV) consisting of a CAM, rotational links with rollers, and torsion springs is proposed. The SJM-IV has the advantage of nonlinear stiffness, which can be achieved only with passive mechanical elements. Various analyses and experiments on static and dynamic collisions show high stiffness of the SJM-IV against an external torque less than a predetermined threshold torque, with an abrupt drop in stiffness when the external torque exceeds this threshold. The safe joint mechanism enables a robot manipulator to guarantee positioning accuracy and collision safety, and which is simple to install between an actuator and a robot link without a significant change in the robot's design.
\end{abstract}

\section{INTRODUCTION}

As robots work in human environments, physical human - robot interaction safety issues have become increasingly important. Several types of safe robot weapons were therefore proposed to improve the safety of collision between humans and robots.

A safe robot arm can be obtained either through a passive or active compliance system. In an active arm, various types of sensors detect a collision and the arm stiffness is controlled adequately by the joint motors. As the active compliance-based approach involves sensing and action in response to dynamic collision, it suffers from relatively low bandwidth unless sophisticated and costly sensors and controllers are used. In addition, the installation of a sensor and actuator in the robot arm often leads to high costs, increased system size and weight, possible sensor noise and malfunction of the actuator.

In contrast, a robot arm based on passive compliance usually consists of completely mechanical elements such as a spring and a flexible connection that can absorb excessive collision. Since this approach uses no sensor or actuator, it can provide fast and reliable responses even for dynamic collision. Several security mechanisms were developed using the passive compliance method.

The spring is by far the most popular mechanical element for the absorption of shock. The soft spring used in the robot arm, however, results in the positioning of inaccuracy because it works even for small external forces that do not require shock absorption. This inaccuracy is

† Corresponding Author, jbsong@korea.ac.kr

(C) 2018 Korea University Mechanical Engineering often made worse by unwanted oscillations caused by the elastic spring behavior. Although a stiff spring can provide high positioning accuracy for a robot arm, its shock absorption capacity is much lower than that of a flexible spring, increasing the probability of injury in the event of a collision with humans. An ideal safe manipulator would therefore exhibit very low stiffness when a collision force could cause human injury but would otherwise maintain very high stiffness.

In previous research done by the Intelligent Robotics Laboratory in Korea University, the safety of a robot arm's joint has been achieved by the safe link mechanism (SLM) and the three versions of the safe joint mechanism (SJM-I, II, III) which only used fundamental mechanical and kinematic elements.

This is the fourth version of the safe joint mechanism (SJM-IV), which removes the linear guide from the previous version (SJM-III) while maintaining functionality, efficiency and compatibility. The main reason for removing the linear guide is to increase financial efficiency. The linear guide in the previous version of the safe join mechanism is easily the costliest component in the design. This easily accumulates when the robot arm has multiple joints that need a safety mechanism implemented.

SJM-IV has only three parts, a pair of torsion springs, a Specially designed CAM, and a pair of spring housings with rollers. The torsion springs are used to absorb shock, and the spring housings with rollers uses the springs' initial torque from the pre-compression to hold the CAM in place in turn allowing the robot arm to function normally under a specified threshold torque. If the robot arm encounters an opposing torque larger than the specified threshold torque, the mechanism triggers 
instantly in response to the excess torque encountered. This mechanism's objective is to guarantee collision safety of a robot arm using simple, fundamental and lowcost mechanical elements without sacrificing the positioning accuracy of the arm.

In this paper, we will be discussing the design element of the new safe joint mechanism in section II, then we will move on to the calculation process and design application in section III followed by a conclusion in section IV.

\section{DESIGN PRINCIPLE OF THE SAFE JOINT MECHANISM-IV}

To simultaneously achieve collision safety and positioning accuracy of a robot arm, we need a nonlinear stiffness mechanism whose stiffness remains very high when the external torque acting on the joint of a manipulator is within the range of normal operation but drops rapidly when the external torque exceeds a certain level due to collision with the object. In this study, a pair of rotational links with pre-compressed torsion springs are exploited to achieve this nonlinear stiffness feature.

\section{II.1 Design}

The mechanism consists of the following parts: Base platform, specially designed CAM, spring housings (rotational links with rollers), CAM followers, Housing caps and torsion springs. The mechanism is represented in the Fig.1.

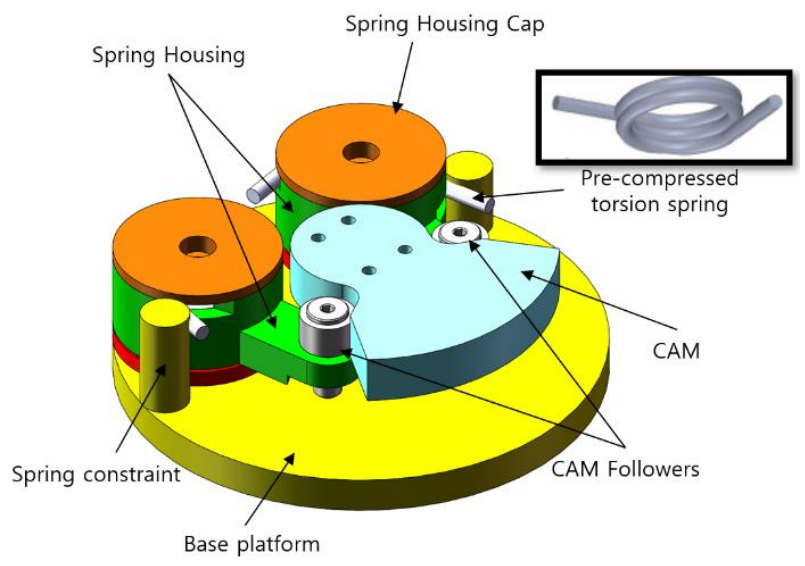

Fig. 1 Safe joint mechanism-IV internal mechanism representation

The base platform is centered with a hole that fits the bearing that connects the CAM to the platform to allow free rotation only in the relevant plane and constrict all other motion. The base platform also has two rods machined into it that constrict the free movement of the torsion springs and allow for a precompression constraint on the springs. The torsion springs are sheltered by housings that also act as objects to transfer the force of said springs. The housings are attached by bearings to a piece attached to the base. This allows free rotation of the housing in reaction to spring's force. The housings also extend with a link of specific length that ends with a half circle centered by rollers or CAM followers that interact directly with the previously mentioned CAM on the surface of thickness. The housing caps act as constraints attached to the base to prevent the torsion springs movement in the normal vector to the base and in turn contains their motion to tension and compression.
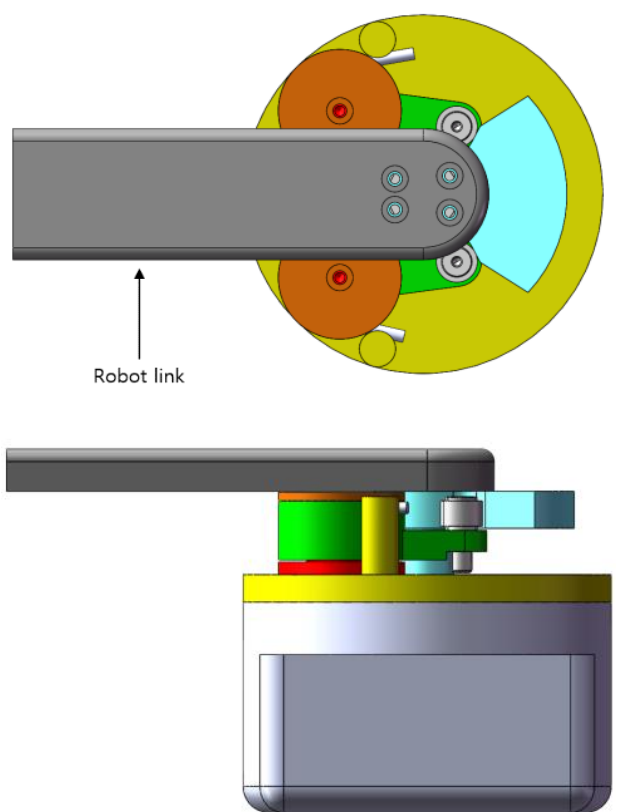

Fig. 2 Safe joint mechanism-IV integrated representation

Fig.2 represents the safe joint mechanism integrated in an example of a robotic joint consisting of a base handle attached to the base platform and a robot link which connects to the CAM directly and is the main source of torque that the mechanism reacts to. The design consists of simple and fundamental mechanical elements that work interactively to insure the stiffness and accuracy of the robot joint while triggering in reaction to a torque exceeding the threshold torque decided by the design of the different components of the mechanism that are going to be discussed in subsection II.2.

\section{II.2 Calculation}

One of the advantages of the safe joint mechanism-IV is the ease of calculation and adaptation of the device in a specific robot joint. Redesigning the joint in accordance with the threshold torque of different joints is less complicated, direct, affects less components in the mechanism and is inexpensive to achieve. 


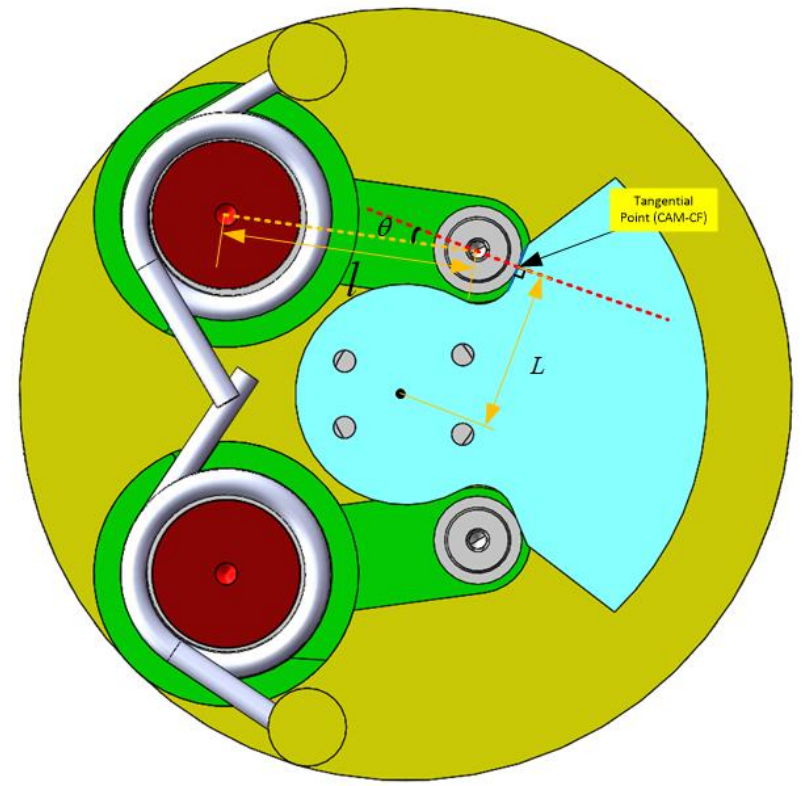

Fig. 3 Safe joint mechanism-IV design terminology

The variable terminology we will be using in this subsection is as follows: $\boldsymbol{K}$ for the torsion spring stiffness, $\boldsymbol{\alpha}$ for the precompression angle, $\boldsymbol{T}_{\boldsymbol{t} \boldsymbol{h}}$ represents the threshold torque that is consistent with the payload of the robot, $\boldsymbol{L}$ is the normal distance between the center of rotation of the CAM and the tangential point of the CAM and CAM follower, $\boldsymbol{l}$ is the distance between the center of the torsion spring and the corresponding center of the CAM follower in the same link. Finally, $\boldsymbol{\theta}$ is the design angle between the centerline of the spring housing link and the normal line perpendicular to the CAM thickness surface at the tangential point between the CAM and roller. Fig.3 shows how what the aforementioned terminology represents in the mechanism's sketch.

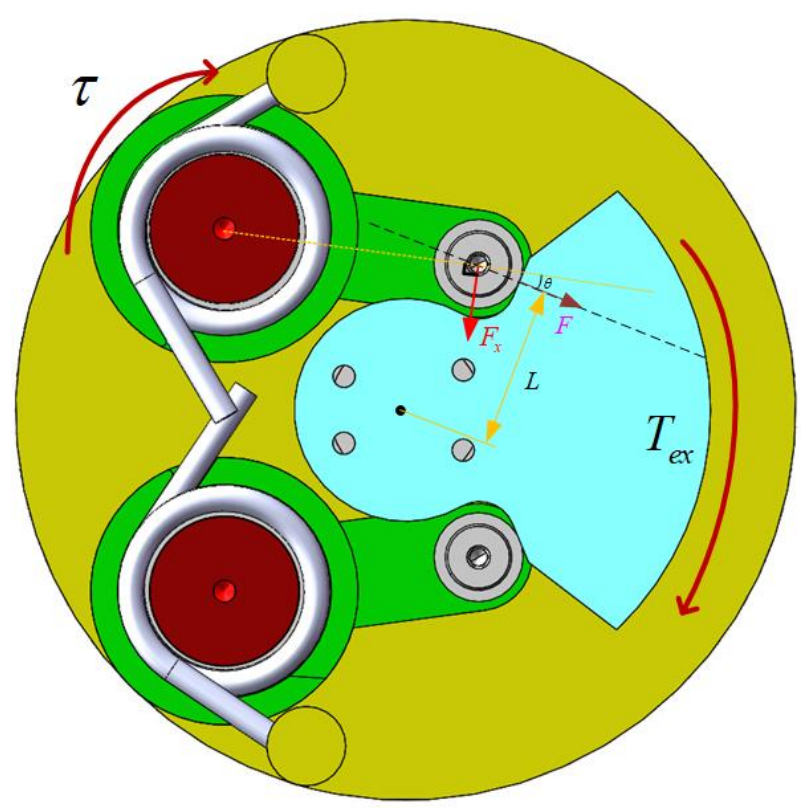

Fig. 4 Safe joint mechanism-IV force and torque representation
In Fig.4, we represent the torque exerted on the spring housing in response to the pre-compression of the spring is represented by $\boldsymbol{\tau}$. We represent the latter with the following equation:

$$
\tau=K \alpha
$$

In turn, the CAM follower exerts a force $\boldsymbol{F}_{\boldsymbol{x}}$ on the $\mathrm{Y}$ axis. Represented by the following equation:

$$
F_{x}=\frac{K \alpha}{l}
$$

The CAM follower exerts force $\boldsymbol{F}$ on the CAM through the tangential point presented in Fig.3. the amount of force depends on the angle $\boldsymbol{\theta}$ between the centerline of the spring housing link and the normal line on the thickness surface of the CAM at the tangential point.

$$
\begin{gathered}
F_{x}=F \sin \theta \\
F=\frac{F_{x}}{\sin \theta} \\
\Rightarrow F=\frac{K \alpha}{l \sin \theta}
\end{gathered}
$$

Through $\boldsymbol{F}$ we calculate the threshold torque $\boldsymbol{T}_{\boldsymbol{t} \boldsymbol{h}}$ which is the torque required to trigger the mechanism as follows:

$$
T_{t h}=\frac{K \alpha L}{l \sin \theta}
$$

Since a pair of spring housings with two rollers pressing on the $\mathrm{CAM}$ on either side of the mechanism, the joint is stable and remains in place within the limits for any torque value exerted by the payload under the threshold torque. If the robot arm encounters a higher torque by colliding with an outside object, the mechanism triggers by rotating the CAM and sliding the roller over the edge of the machined cut extrusion. This allows the force from the collision to be absorbed by the torsion spring and gradually decreasing angle $\boldsymbol{\theta}$ caused by the rotation to dampen the force. The rotation angle is limited by the cut out on the spring housing to constrain the torsion spring as presented in Fig.5.

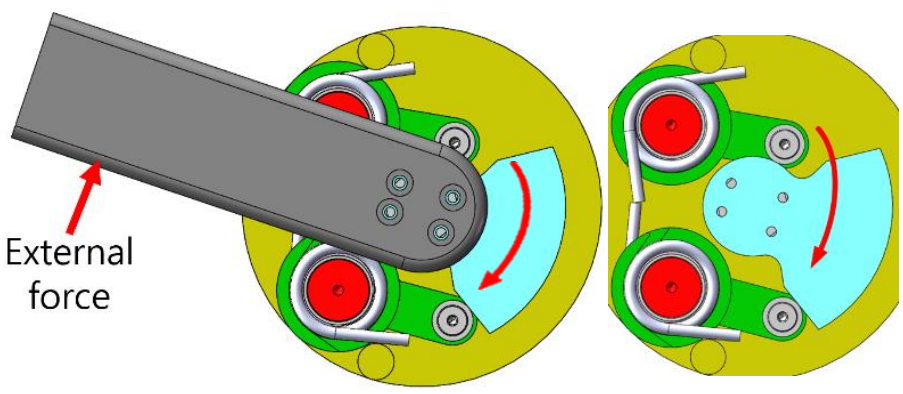

Fig. 5 Safe joint mechanism-IV trigger representation 


\section{EXPERIMENTATION OF SAFE JOINT MECHANISM-IV}

\section{A. Experimental Setups}

To verify the functionality of the safe joint mechanism$\mathrm{IV}$, the mechanism was manufactured according to the original design with a threshold torque of $T_{t h}=8 \mathrm{~N} \cdot \mathrm{m}$. The spring stiffness $\mathrm{K}$, pre-compression angle $\alpha$, distance between the tangential point and CAM center of rotation L, distance between the center of the torsion spring and roller $l$ and angle of CAM incline $\theta$ were set to $76.3 \mathrm{~N} \cdot \mathrm{mm} / \mathrm{deg}, 20$ deg, $18 \mathrm{~mm}, 24 \mathrm{~mm}$ and $30 \mathrm{deg}$ respectively. An M4 hole was tapped in the robot link with a $8.2 \mathrm{~mm}$ distance from the CAM center of rotation.

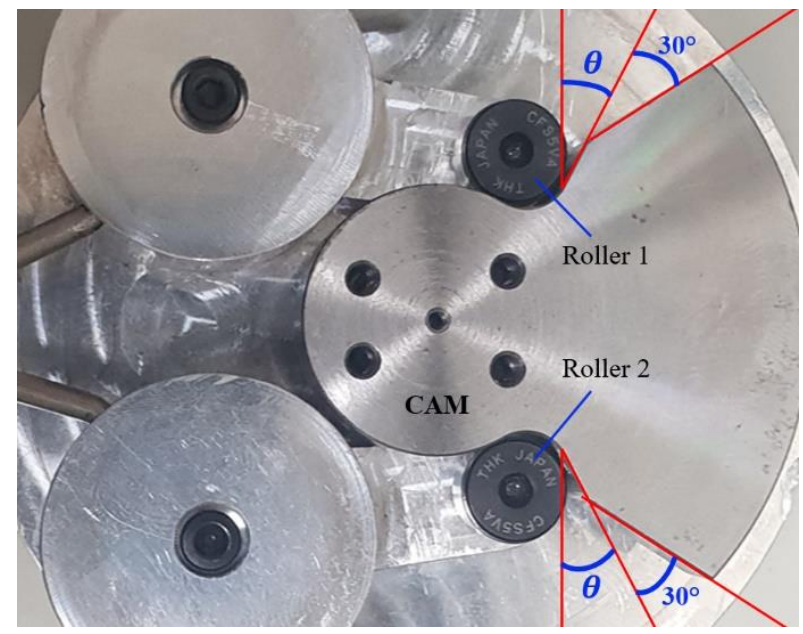

Fig. 5 Symmetric CAM of SJM-IV

\section{B. Experimental Results}

An electronic weight scaler was used to exert force on the M4 bolt screwed to the tapped hole on the robot link. Through that, the torque exerted on the mechanism was measured as shown in Fig. 6. From Fig. 6 (a) it is obvious that the SJM-IV maintains the robot arm's normal function as long as it is functioning under the threshold torque. When the torque exceeds the set threshold (in this case $2.5 \mathrm{~kg}$ ), the mechanism triggers and the torsion spring on either side absorbs the shock of the collision. When the maximum threshold torque is exceeded, the robot link will rotate for a maximum of 30 degrees while absorbing the shock relatively to the handle or the link attached on the other side of the joint as represented in Fig. 6 (b). There is still room for development of the mechanism that allows it to absorb shock for a larger angle. Also depending on the size of the mechanism, there might be limitation for the components available on the market such as the torsion spring or the material the mechanism is made of.

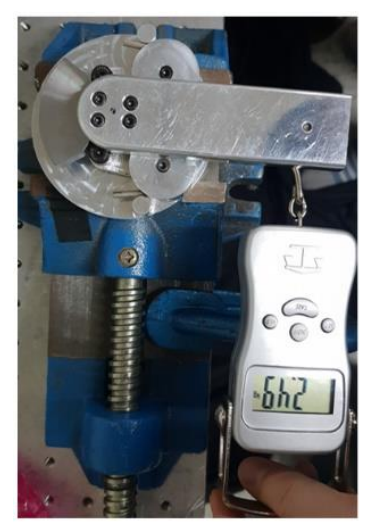

(a)

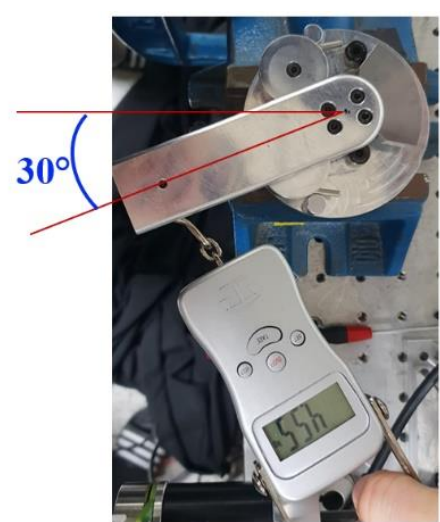

(b)
Fig. 6 Representation of the SJM-IV functioning before and after exceeding maximum threshold torque

In another experiment for static collision of the SJM, the end-point of the robot arm was initially positioned to barely touch a fixed plate, and the joint torque provided by the motor was slowly increased. The static collision force between the robot and the fixed plate was measured by a force/torque sensor. As shown in Fig. 7, experiments were conducted for the robotic arms with and without the SJM. The robot link was forced to rotate in both the direction of gravity and the opposite direction.

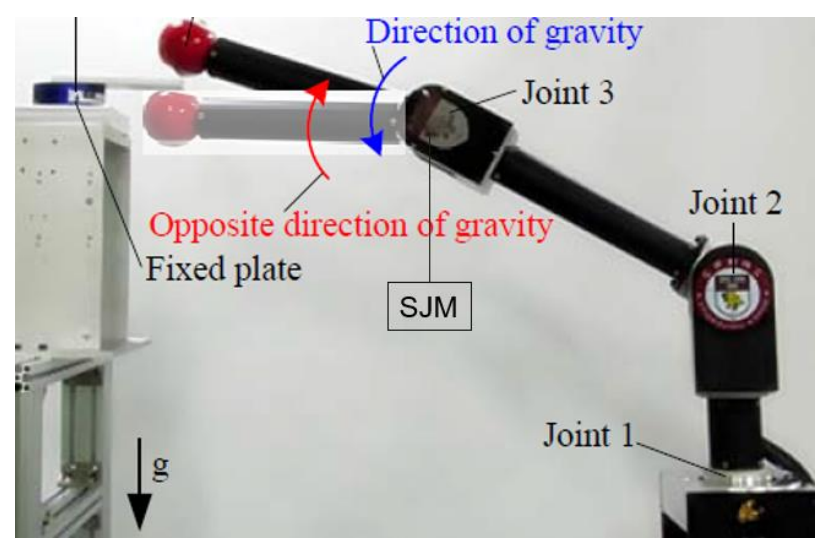

Fig. 7 Experimental setup for static collision

As shown in Fig. 8, virtually no displacement of the SJM occurred in the opposite direction of gravity when external torque due to the contact force was below $8 \mathrm{~N} \cdot \mathrm{m}$. Therefore, the robot arm with the SJM can accurately handle a payload of up to approximately $1.5 \mathrm{~kg}(15 \mathrm{~N})$ as though it had a speed reducer with a high gear ratio. However, as the external torque increased above the threshold torque, the stiffness of the SJM quickly diminished. Thus, the SJM provided high positioning accuracy of the robot arm in normal operation and guarantees safe human-robot contact by absorbing a contact force above $50 \mathrm{~N}$ in a collision. 

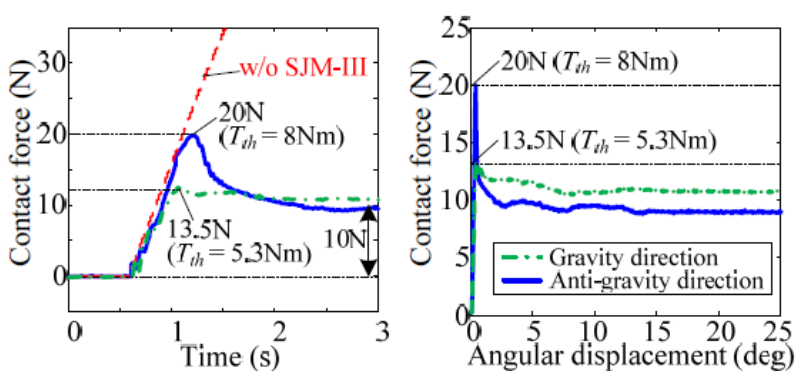

Fig. 5 Experimental results for static collision of robot arm.

\section{CONCLUSION}

In this study, the fourth version of a safe joint mechanism (SJM-IV) was proposed for collision safety. SJM-IV is simpler, has a lower cost, higher torque resistance ability and more adaptability than the previous SJMs. A robot arm equipped with the SJM-IV can maintain very high stiffness up to a preset threshold torque and provides a very low stiffness above the threshold. From our analysis and experiments, the following conclusions are drawn:

1) The stiffness of the robot manipulator abruptly drops if the external torque to the SJM-IV exceeds the pre-determined threshold torque. Therefore, collision safety can be achieved even for a high-speed dynamic collision.

2) High stiffness of the robot arm can be maintained for external torque that is lower than the threshold torque. Therefore, positioning accuracy can be achieved in normal operation.

4) The SJM-IV can be designed in consideration of gravity compensation by setting different threshold torques in both directions of rotation.

5) Since a collision is detected by limit switches, a robot arm with the SJM-IV can react to the collision. Therefore, the problem with the limit of the operating range of the SJM-IV can be solved, thus maintaining the robot arm in the safe region.

6) Since the SJM-IV is simple enough to be installed between an actuator and a robot link, it can be applied to any type of robot arm without much modification done to the robot.

Currently, simpler and more lightweight safe joint mechanisms are under development so that several SJMs may be used simultaneously in the robot arm without a significant increase in cost and weight.

\section{REFERENCES}

[1] Jung-Jun Park, Jae-Bok Song, 2010, "Safe Joint Mechanism using Inclined Link with Springs" IEEE/RSJ International Conference on Robotics and Automation.

[2] Hwi-Su Kim, In-Moon Kim, Chang-Nho Cho, JaeBok Song, 2012, "Safe joint module for safe robot arm based on passive and active compliance method" 2012 Elsevier Ltd. All rights reserved.

[3] Hwi-Su Kim, Jung-Jun Park, Jae-Bok Song, 2008, "Safe Joint Mechanism using Double Slider Mechanism and Spring for Humanoid Robot Arm" IEEE/RSJ International Conference on Robotics and Automation.

[4] 송재복, 김병상, 2011. 유연 운동을 위한 가변 강성 액츄에이터. 로봇과 인간, $8(1), 3-11$. 